\title{
Optical biosensor based on the dependent expression of fluorescent proteins
}

\author{
Janette Kothe ${ }^{1}$ (janette.kothe@mailbox.tu-dresden), Anna Schröter ${ }^{1}$, Kristof Zarschler ${ }^{2}$, Dagmar Wersing ${ }^{3}$, Martin \\ Mkandawire $^{3}$, Gerald Gerlach ${ }^{1}$, Gerhard Rödel ${ }^{2}$, Wolfgang Pompe ${ }^{3}$ \\ ${ }^{1}$ Solid-State Electronics Laboratory, Technische Universität Dresden \\ 2 Institute of Genetics, Technische Universität Dresden \\ ${ }^{3}$ Institute for Material Science, Technische Universität Dresden \\ 01062 Dresden, Germany
}

\section{Introduction}

There are many measurement tasks in process engineering that cannot be solved with conventional chemical or physical methods. As an example, concentration measurements in chemical production or biotechnology usually need expansive analysis equipment which needs manual handling and extensive sample preparation in a laboratory environment, due to a lack of reliable and long-term stable in-line or on-line sensors ([1], [2], [3]).

With the ongoing development of biological assays these transducers become a promising candidate for the design of a new sensor class that fulfills the common requirements concerning robustness and reliability. In comparison to conventional physical and chemical analysis technologies there are some advantages that have to be considered:

- Biological transducers rely on bio-chemical reception mechanisms, which feature good selectivity to certain measurands while giving the possibility to react on several inputs differently in the sense of a multi-parameter sensor.

- Biological transducers with their specifically binding receptors are working with a magnificently high sensitivity, down to single-molecule detection. On the other hand they provide a high flexibility regarding their detection range by self-controlled adaption mechanisms.

If one succeeds in integrating those biological components in the typical system architectures of process control, new application fields will be accessible - even beyond a pure transferring of lab equipment to the field. In the development of on-line biosensors the assays have to fulfill certain preconditions:

- The assay should work multiple times.

- The assay should work without additional reagents that would have to be fed to the sensor under using of complex sample handling systems.

- There has to be a technological possibility to immobilize the biological transducer reliably on technical surfaces.

- The assay has to be long-term stable and robust to endure in industrial environments without a decay of its sensor function.

- The assay reaction should be as fast as possible to afford the integration in process control protocols.

This paper will consider whole-cell sensors that use the advantages of biological assays mentioned before and that enable at least on-line integration within the process line.

\section{Whole-cell Sensors}

Whole-cell sensors are living biological systems using the reception function of microorganisms to realize sensor functions ([4], [5]). They comprise microorganisms like yeasts or bacteria, which are genetically modified, so that they react on a configurable stimulus, e.g. a heavy metal concentration in the measurement medium with a dependent fluorescence. The receptors of the cells detect specific signal molecules and stimulate the organism to produce a fluorescent protein. This dependent expression can be increased by using quorum sensing in cellular amplifier systems (Fig. 1). Sensor cells react on the presence of a defined stimulus with the production of a small soluble peptide, the yeast pheromone ( $\alpha$-factor). 
These secreted molecules are disseminated to other cells that thereby start expressing a fluorescent protein, e.g. green fluorescent protein (GFP [6]) (Fig. 2).

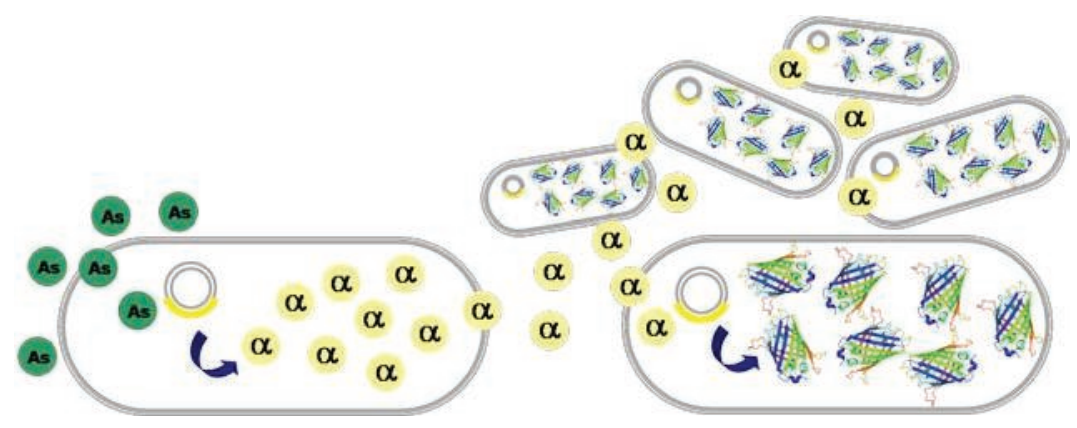

Fig. 1: Scheme of a cellular amplifier system: sensor cells react on the presence of a configurable stimulus as a certain arsenic concentration with the production of $\alpha$ promoters. These promoters are disseminated to cells that thereby start expressing the fluorescent protein.

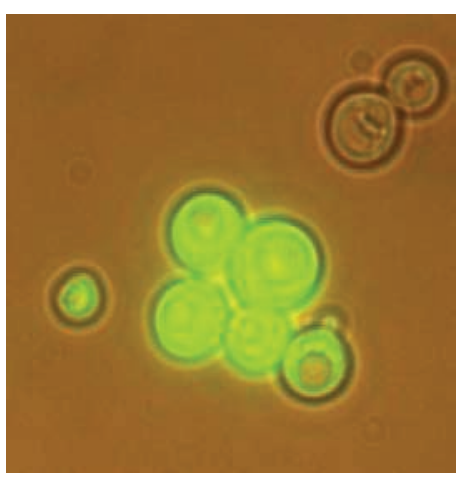

Fig. 2: Expression of fluorophors: Microscopic image of yeast cells (S.cerevisiae) that constitutively produce the enhanced green fluorescent protein (eGFP).

For the proof of principle a whole-cell sensor is built up, which bears on the dependent expression of GFP by a cellular amplifier system of yeast S.cerevisiae. It detects the concentration of arsenic in aqueous solutions. Beside the biological transducer a compact optical sensor for the acquisition of the fluorescent emission integrates the excitation source, a fluidic system and an optical detector in an industrial standard package. With the design of this integrated detection system the potential for a robust, optical biosensors will be investigated.

\section{Methods}

The evaluation of the GFP concentration depending on the arsenic concentration is based on the optical detection in a particular range of wavelengths. This may be realized either by a reflective, evanescent or transmissive arrangement. To provide a very compact design with high optical efficiency a transmissive topology seems to be favorable (Fig. 3). Compared to alternative topologies it provides the following advantages:

- $\quad$ easy to integrate in a system-in-package

- High geometry factors

- Adaptable to other optical outputs of cholorimetric assays, absorption assays or bioluminescent assays

Admittedly, such a topology needs optical filters for the separation of the absorption wavelengths of the fluorophore from the fluorescent emission wavelengths. To keep the complexity of the optical system low, only one filter is used. Light emitting diodes (LED) with a central wavelength of $465 \mathrm{~nm}$ are used as an excitation source for the fluorophor. The optical detector is a silicon $\mathrm{p}-\mathrm{i}-\mathrm{n}$ diode (Silicon Sensor PC10-6b) with a detection area of $10 \mathrm{~mm}^{2}$.

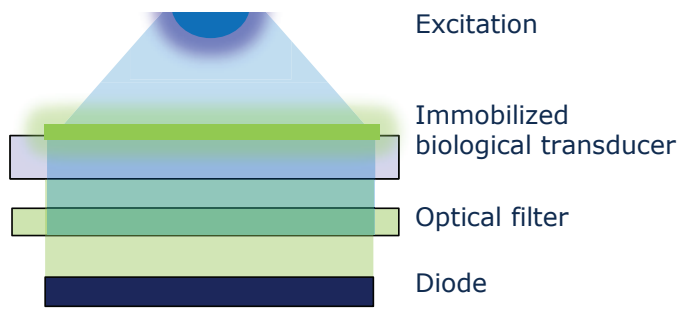

Fig. 3: Transmissive topology for detecting bio-fluorescence 
Fig. 4 describes schematically the operation of the fluorescence measurement system.

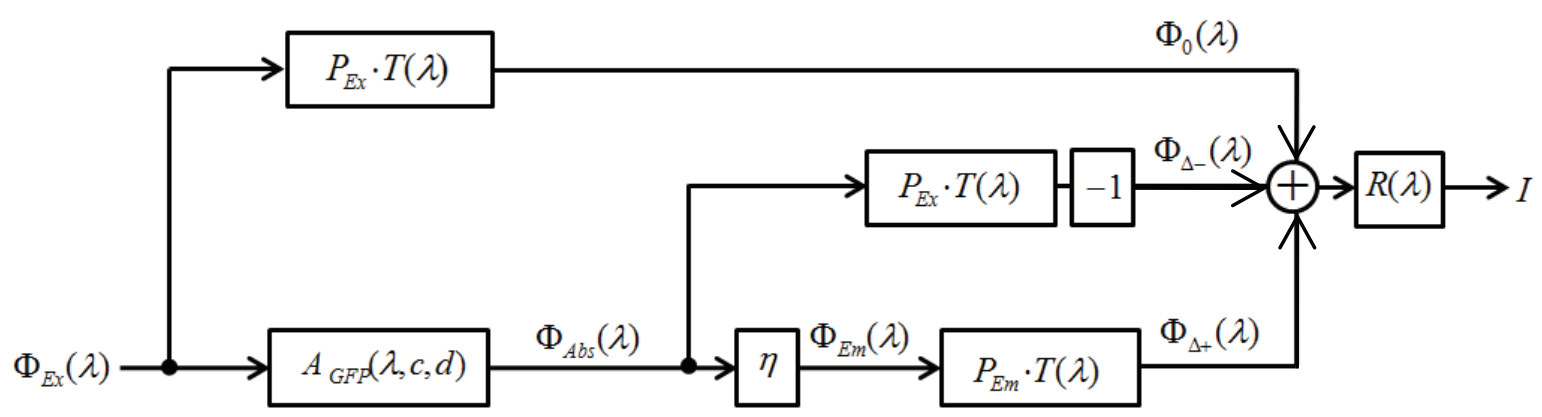

Fig. 4: Modell of the fluorescence detection system

\begin{tabular}{|c|c|c|c|}
\hline$A_{G F P}(\lambda, c, d)$ & $\begin{array}{l}\text {... Absorption of the fluorophore, } \\
\text { distinguished by its concentration and the } \\
\text { thickness of the sample }\end{array}$ & $\eta$ & $\ldots$ Quantum yield of the fluorophore \\
\hline$I$ & $\ldots$ Reverse photocurrent & $\lambda$ & $\ldots$ wavelength \\
\hline$P_{e m}$ & ... Geometry factor of emission & $\Phi_{0}(\lambda)$ & $\begin{array}{l}\text {... Radiant offset, corresponding to leackage of } \\
\text { excitation radiation at the detector }\end{array}$ \\
\hline$P_{e x}$ & ... Geometry factor of excitation & $\Phi_{\Delta_{-}}(\lambda)$ & $\begin{array}{l}\text {... Negative radiant absorption signal at the } \\
\text { detector }\end{array}$ \\
\hline$R(\lambda)$ & $\ldots$ Responsivity of the detector & $\Phi_{\Delta_{+}}(\lambda)$ & ... Radiant emission signal at the detector \\
\hline \multirow[t]{3}{*}{$T(\lambda)$} & ... Transmission of the optical filter & $\Phi_{a b s}(\lambda)$ & ... Absorbed radiant flux \\
\hline & & $\Phi_{e m}(\lambda)$ & ... Emitted radiant flux \\
\hline & & $\Phi_{e x}(\lambda)$ & ... Exciting radiant flux \\
\hline
\end{tabular}

A part of the radiant power $\Phi_{e x}$ of the LED will be absorbed by the fluorophore $\left(\Phi_{a b s}\right)$, another unwanted portion does not affect the fluorescence and leads to a radiant offset $\Phi_{0}$. The absorbed radiant flux $\Phi_{a b s}$ is distinguished by the extinction characteristic $A_{G F P}$ of the fluorophore. Coupled by the quantum yield $\eta$ it generates the fluorescent emission $\Phi_{e m}([7],[8])$

Whether it is possible to detect the fluorescence depends on the ability of the filter $T(\lambda)$ to exclude the absorption while permitting the emission to pass, and accordingly minimizing the negative absorption signal $\Phi_{\Delta_{-}}$and maximizing the positive radiant signal of emission $\Phi_{\Delta_{+}}$. Also the geometric factor of excitation $P_{e x}$ has an influence on the absorption signal $\Phi_{\Delta_{-}}$. This factor describes the fraction of exciting radiation that reaches the detector due to topology of the system. In a transmissive setup it will be near to unity, hence it cannot be used to oppress the absorption signal $\Phi_{a b s}$ and the excitation offset $\Phi_{0}$. The geometric influence on the emission is described by the geometry factor of emission $P_{e m}$, which describes the fraction of fluorescent radiation that reaches the detector. Due to the diffuse characteristic of the fluorescent emission it becomes an important figure of merit for the measurement system. The three radiant channels sum up to one signal that is converted to a photocurrent $I$ with the responsivity $R$ of the detector.

\section{Modelling and Simulation}

Figs. 5 to 7 present the simulation results on the prospective performance of the transmissive measurement setup. Fig. 5 shows simulation results for typical radiation fluxes of excitation, absorption and emis- 
sion in the detection setup without a filter influence. A characteristic of biological fluorophores is their weak extinction compared to synthetic dyes. This diminishes absorption of the excitation source leading to an accordantly weak emission.

The geometric factor of emission determines the spectral intersection point of absorption and emission of the fluorophore and thereby the ideal border between blocking and transmission interval for a longpass filter. The use of a bandpass filter gives the additional possibility to detect certain fluorophores with high selectivity and to distinguish between two fluorophores. This provides the possibility to design multiparameter sensors, in which the biological transducer reacts on different signal molecules with the expression of different fluorophores.

While the transmission range of an ideal filter would start at $505 \mathrm{~nm}$, the simulation displayed in Fig. 6 uses the characteristics of a commercially available filter (Edmund Optics Longpass 500, OD4). Fig. 7 depicts the resulting current signal that results from absorption and emission influence. Due to the nonoptimal performance of the filter there is a high leakage of the negative absorption signal.

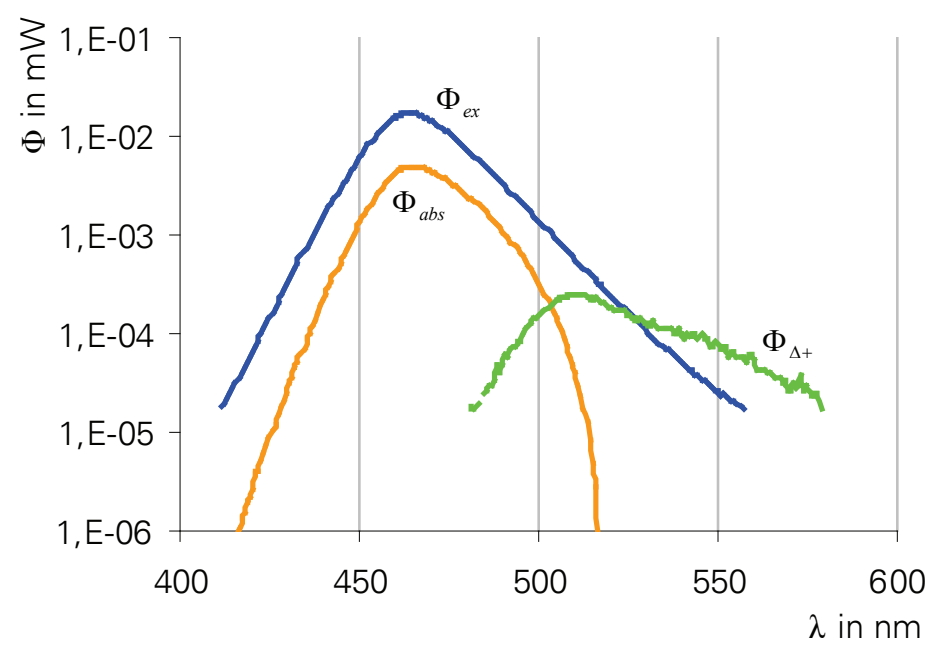

Fig. 5: Simulated radiant fluxes in the fluorescence detection setup without a filter:

Excitation $\Phi_{e x}$ (blue), absorbed radiation $\Phi_{a b s}$ (orange), fluorescence emission signal $\Phi_{\Delta+}$ (green)

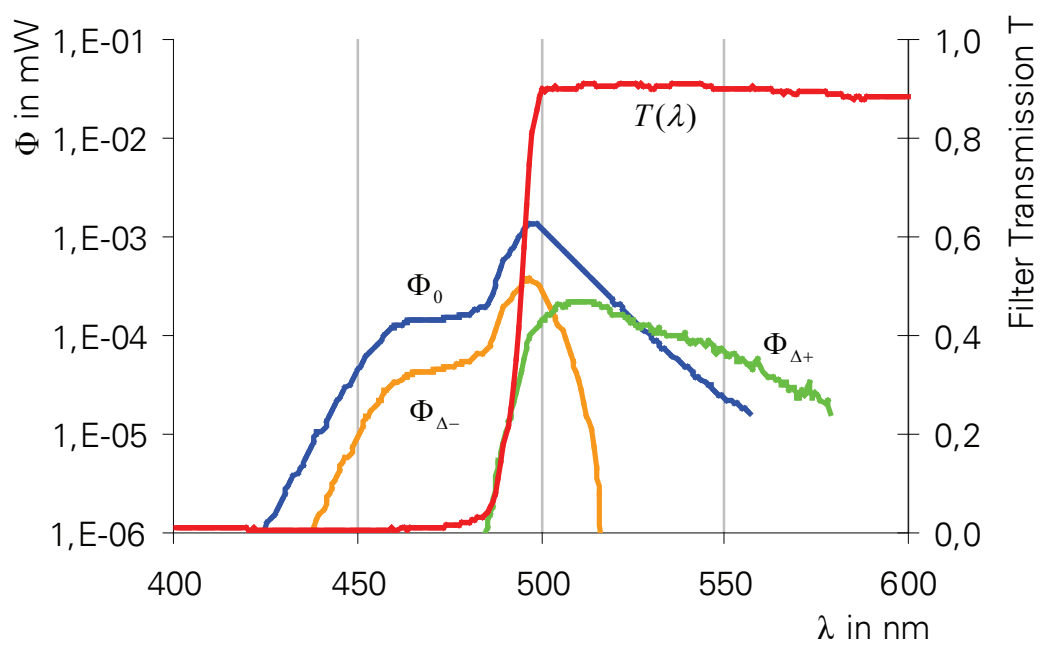

Fig. 6: Calculated effect of a longpass filter (red) on the radiation in the fluorescence detection setup 


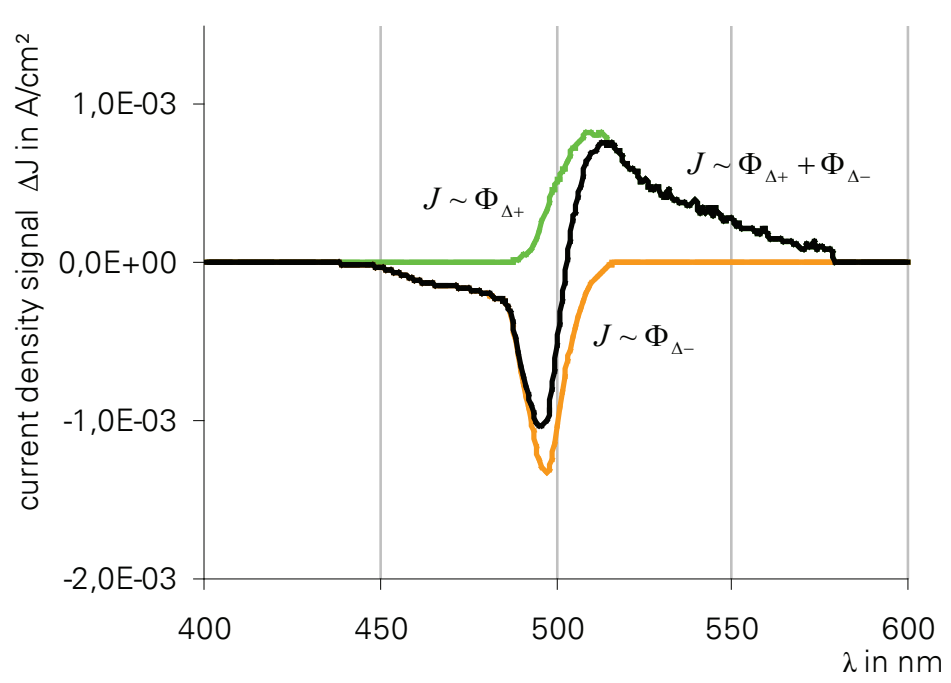

Fig. 7: Caculated resulting current signal at a typical $p-i-n$ diode detector

\section{Experimental Results}

Fig. 8a shows the concentration characteristics of the fluorescence detection system obtained from experimental investigations. The reason for the high offset in the reverse current of the $p$-i-n diode detector is given by the wider spectral configuration of the LED sources, respectively the missing excitation short pass filter. The geometry factor of emission was calculated by simulation to be approximately $8 \%$.

Fig. 8b compares a sample of wild type yeast with cells that constitutively express GFP. It could be shown that it is possible to detect the bio-fluorescence of the biological transducers with the transmissive topology from the arrangement in Fig. 3.

a)

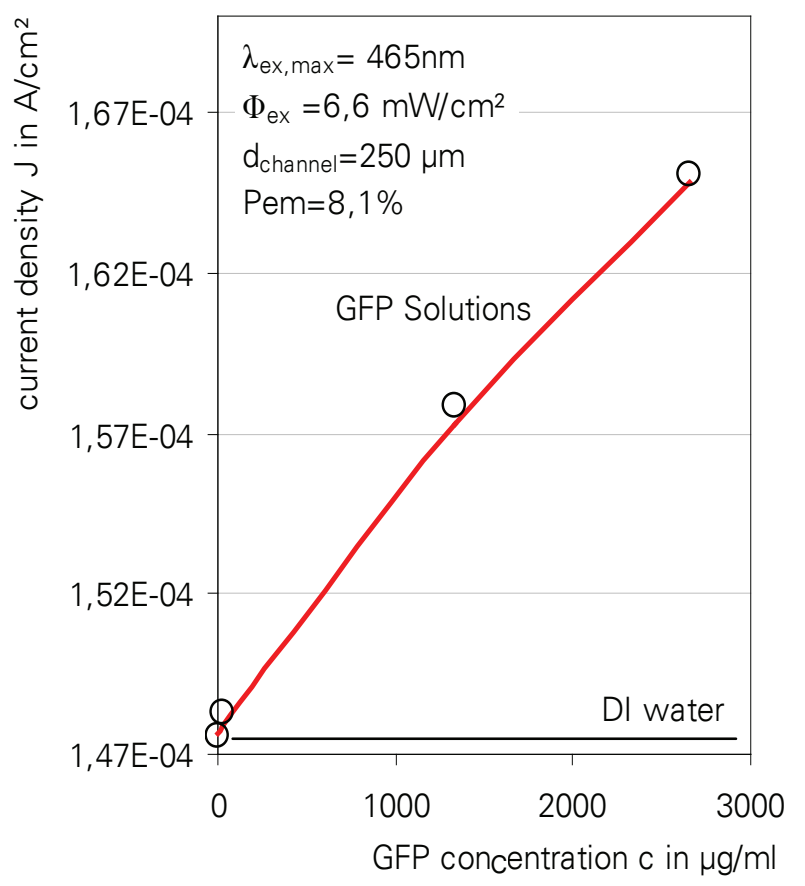

b)

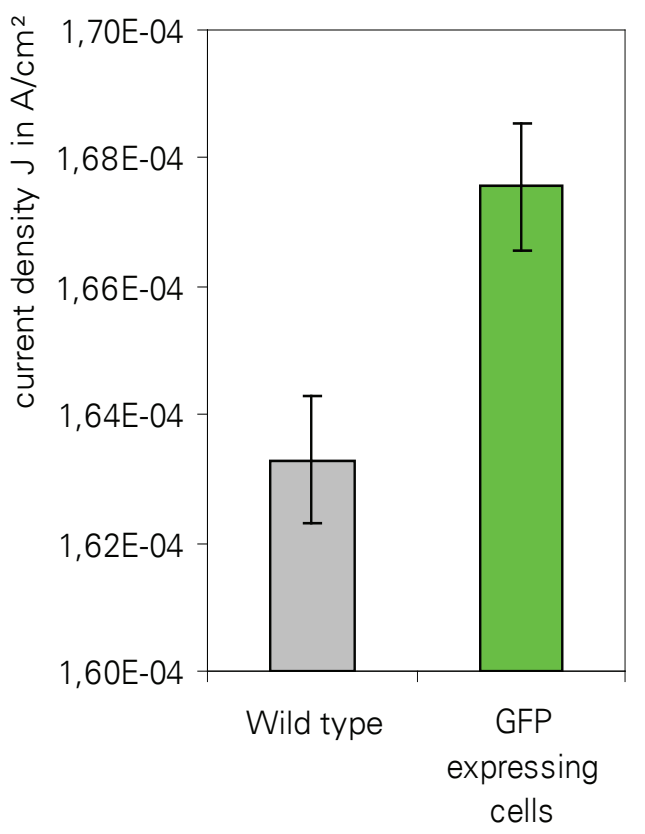

cell concentration OD 1,6

Fig. 8: Output signal of a whole-cell sensor:

a) GFP concentration characteristic: --- simulated; o measured

b) Detection of bio-fluorescence of GFP expressing yeast cells 


\section{Conclusions and Outlook}

Both simulation and experimental results gave evidence that bio-fluorescence can be reliably be detected with a setup comprising a LED as source for exciting a fluorescence, a photodetector and GFP expressing cells as well as a single optical filter in a transmissive arrangement. This enables to build up a compact and robust optical biosensor for near-line process control. Next steps will include optimization of the setup, integration of all components in one package and investigations on long-term stability.

\section{Acknowledgements}

The presented results were achieved within the project "ChiBs - Chip-basierte Biosensor-Systeme" that was founded by the German Federal Ministry of Education and Research (BMBF). The authors also acknowledge the support of the Research Training Group "Nano- and Biotechnologies for the Packaging of Electronic Systems" (DFG GRK 1401).

\section{References}

[1] Kessler, Rudolf W. (Hg.) (2006): Prozessanalytik. Strategien und Fallbeispiele aus der industriellen Praxis. Weinheim: Wiley-VCH, p.16

[2] McMahon, Gillian (2007): Analytical instrumentation. A guide to laboratory, portable and miniaturized instruments. Chichester: Wiley, p.4-6

[3] Valcarcel, Miguel; Castro, Maria Dolores Luque de (1993): Flow-through (bio)chemical sensorsPlenary lecture. In: Analyst, Jg. 118, H. 6, p. 593-600.

[4] K.Ostermann, W.Pompe, H.Böttcher, G.Rödel, A.Gross (2008): Whole-Cell Sensor. International Patent WO002008132178A1

[5] K.Ostermann, W.Pompe, D.Wersing, M.Mertig, J.Gooding, G.Rödel (2006): Verfahren und Vorrichtung zur Detektion von Mikroorganismen und/oder deren Aktivität. German Patent DE102006057975B4.

[6] R.Tsien (1998): The green fluorescent protein. Annual Review of Biochemistry, Jg. 67, H. 1, p. 509-544.

[7] Mielenz, Klaus D (1982): Measurement of photoluminescence. New York, N.Y.: Acad. Pr. (Optical radiation measurements, 3 )

[8] E. Goldys (2009): Fluorescence applications in biotechnology and life sciences. Wiley-Blackwell. 